\title{
Déjà vu: Ralstonia mannitolilytica infection associated with a humidifying respiratory therapy device, Israel, June to July 2011
}

C Block (colinb@ekmd.huji.ac.il)1, Z Ergaz-Shaltiel ${ }^{2}$, L Valinsky³, V Temper ${ }^{1}$, C Hidalgo-Grass ${ }^{1}$, N Minster ${ }^{1}$, C Weissman $^{4}$, S Benenson ${ }^{1}$, J Jaffe ${ }^{3}$, A E Moses ${ }^{1}$, B Bar- $-z^{2}$

1. Department of Clinical Microbiology and Infectious Diseases, Hadassah-Hebrew University Medical Centre, Jerusalem, Israel

2. Department of Neonatal Medicine, Hadassah-Hebrew University Medical Centre, Jerusalem, Israel

3. Laboratory of Molecular Biology, Israel Ministry of Health, Jerusalem, Israel

4. Department of Anaesthesiology, Hadassah-Hebrew University Medical Centre, Jerusalem, Israel

Block C, Ergaz-Shaltiel Z, Valinsky L, Temper V, Hidalgo-Grass C, Minster N, Weissman C, Benenson S, Jaffe J, Moses AE, Bar-Oz B. Déjà vu: Ralstonia mannitolilytica infection associated with a humidifying respiratory therapy device, Israel, June to July 2011. Euro Surveill. 2013;18(18):pii=20471. Available online: http://www.

eurosurveillance.org/ViewArticle.aspx?Articleld $=20471$

Article submitted on 20 July 2012 / published on 2 May 2013

Following a bloodstream infection in June 2011 with Ralstonia mannitolilytica in a premature infant treated with a humidifying respiratory therapy device, an investigation was initiated at the Hadassah Medical Centres in Jerusalem. The device delivers a warmed and humidified mixture of air and oxygen to patients by nasal cannula. The investigation revealed colonisation with $R$. mannitolilytica of two of 15 patients and contamination of components of five of six devices deployed in the premature units of the Hadassah hospitals. Ten isolates from the investigation were highly related and indistinguishable from isolates described in an outbreak in 2005 in the United States (US). Measures successful in containing the US outbreak were not included in user instructions provided to our hospitals by the distributor of the device.

\section{Introduction}

In June 2011, we encountered a case of bloodstream infection due to a Gram-negative bacillus in a premature infant who failed to respond to the initial antibiotic combination of ampicillin, cefotaxime and gentamicin, and later to meropenem. The organism was identified by molecular analysis as Ralstonia mannitolilytica. It proved to be resistant to all the above antibiotics and the child recovered after the treatment was changed to co-trimoxazole. The child was treated with a respiratory therapy device for three weeks, during which time the bloodstream infection developed. $R$. mannitolilytica won some notoriety in an outbreak associated with the use of this device in the United States (US) in 2005 [1]. After an initial investigation by the Centers for Disease Control and Prevention (CDC) revealed deficiencies in the reprocessing of the device at that time, it was recalled by the manufacturer in January 2006 and reintroduced a year later with a revised procedure for decontamination and reprocessing approved by the US Food and Drug Administration (FDA).
Organisms of the genus Ralstonia [2] are aerobic Gramnegative bacilli commonly found in water and other moist environments [3], but isolated infrequently from clinical material, and then mostly in connection with healthcare-associated infections with environmental sources [4]. In recent years Ralstonia spp. have been recognised increasingly in sputum from cystic fibrosis patients [5,6]. The species $R$. mannitolilytica [7] has rarely been reported as a cause of human disease, but has been implicated in clusters related to suspected contamination of medical solutions or equipment $[1,8$ 10] as well as among the Ralstonia spp. in sputum of patients with cystic fibrosis $[7,11,12]$.

The Vapotherm 2000i (Vapotherm, Stevensville MD, US) is a system for delivering a warmed and humidified oxygen-air mixture to patients, mostly infants, via nasal cannulae (Figure 1). The conditioning of the gases occurs in a vapour transfer cartridge. The system was introduced at our medical centre in January 2007, contemporaneously with its reintroduction to the US market following the outbreak of contamination with $R$. mannitolilytica $[1,8,13]$.

The Hadassah Medical Centre comprises two hospitals, one at Ein Kerem, with approximately 750 beds, and the other at Mount Scopus with about 300 beds. Both have Premature Baby Units, with the primary facility located at Mount Scopus. As soon as the identity of the blood culture isolate was established, and in light of the US experience, the four Vapotherm devices in use at Mount Scopus and the two deployed at Ein Kerem were withdrawn from use. An investigation was initiated to determine whether an environmental source could be found for the organism and whether more infants had been colonised or infected. 
Flow diagram of the Vapotherm 2000i system (A) and section of vapour transfer cartridge (B)

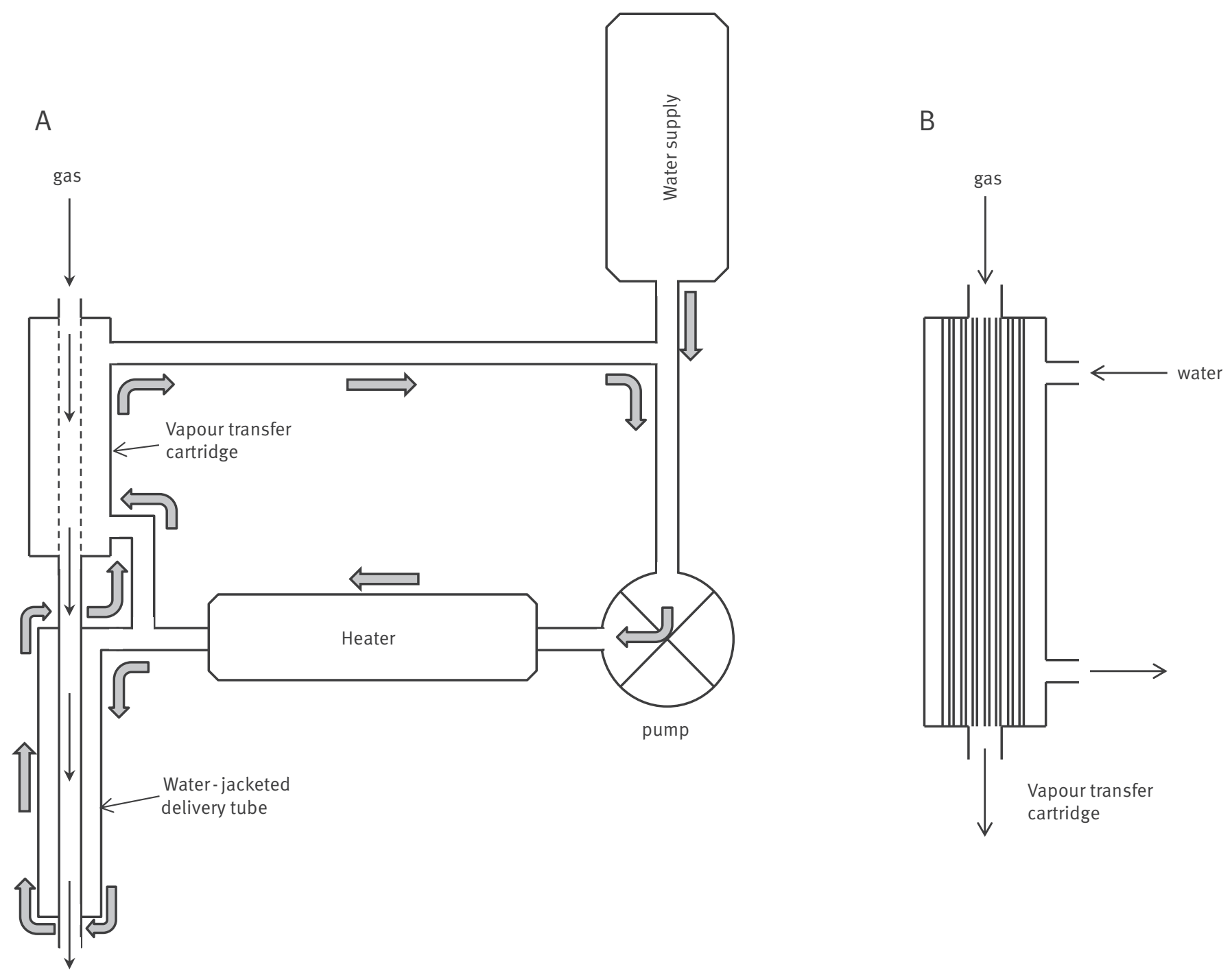

Panel B illustrates the hundreds of hollow fibers through which the gas passes surrounded by the humidifying water.

\section{Methods}

\section{Patient samples}

Fifteen premature infants, including the index patient, were present in the Mount Scopus unit when the aetiological diagnosis of the bloodstream infection was confirmed. Nine of these had been treated at various times with the Vapotherm 200oi. Blood cultures are taken frequently from premature babies, but $R$. mannitolilytica was recovered from blood only from the index patient. Cultures were taken from the nares and oral cavity of all 15 infants.

Blood cultures were performed using the Bactec 9240 system (Becton Dickinson, Sparks MD, US). Nasal and oral cultures were collected from the 15 premature infants hospitalised at Mount Scopus using synthetic sterile swabs transported in Amies's medium (Copan, Brescia, Italy), plated on MacConkey agar and tryptic soy agar (Novamed, Jerusalem Israel), incubated initially at $37^{\circ} \mathrm{C}$ overnight and then at room temperature.

\section{Environmental samples}

Maintenance and reprocessing of the system, including disinfection of the cartridges, were carried out according to instructions provided in Hebrew by the local distributor. Sterile water for the humidification and warming functions of the system was provided in semirigid plastic containers manufactured by the Hadassah Pharmacy Service. Vapour transfer cartridges were disinfected between patients or discarded after two months of use. Cartridges that were used on patients with clinical infection were discarded. All tubing was replaced for each new patient. External cleaning and 
decontamination of the devices was carried out in accordance with local procedures.

Methods for culture of components of the Vapotherm 2000 i system were adapted from those employed by the CDC during the US investigation [1]. External surfaces of the device were not sampled for culture, the focus being placed on the tubing and the humidifying cartridges. Cultures were taken from the Vapotherm delivery tube in use on the index patient. The gas line was sampled under aseptic conditions by flushing with $45 \mathrm{~mL}$ of sterile water into a sterile filtration device designed for microbiological sampling (Millipore Microfil system, Millipore, Billerica MA, US). The filter membrane was placed aseptically on tryptic soy agar (Novamed, Jerusalem, Israel) and incubated as above. The water from the outer sleeve of the delivery tube was removed aseptically and filtered and cultured in the same way.

All six vapour transfer cartridges in use at the time when the devices were removed from service (four from Mount Scopus and two from Ein Kerem) were sampled as for the gas line. The cartridges had been in use for periods of time varying from a few days to three weeks. In addition, three unopened cartridges remained in stock, all from the same batch. A sample from one of these was cultured and the remaining two were aseptically dismantled and attempts made to extract bacterial DNA from their contents for 16S rRNA analysis. The sterile water used for the cultures was the same product used for humidification in the operation of the Vapotherm 2000i. This water was produced at that time in the hospital's pharmacy production facility and provided in sterile $1 \mathrm{~L}$ plastic containers. The contents of two of these containers were cultured during the investigation, by passing the whole fluid volume through the filters.

\section{Identification and typing}

Identification of the index patient's blood culture isolates and the initial environmental isolates from the delivery tube of the index case was attempted using the API 20NE kit (BioMerieux, Marcy l'Etoile, France). Identity was established for all isolates by 16S rRNA gene sequencing [14] followed by sequence comparison using the Basic Local Alignment Search Tool (BLAST) and non-redundant (nr) database from NCBI (http://blast.ncbi.nlm.nih.gov/Blast.cgi). These and all other isolates were then characterised at the Molecular Epidemiology Laboratory of the Israel Ministry of Health by pulsed-field gel electrophoresis (PFGE) using Spel, according to the PulseNet protocol [15]. Comparison with the isolates of $R$. mannitolilytica from the US outbreak in 2005 was performed bilaterally by the Ministry of Health laboratory and the US CDC, using the BioNumerics 6.5 software package (Applied Maths NV, Sint-Martens-Latem, Belgium) with dice coefficients, a $1 \%$ position tolerance and optimisation values. Cluster analysis was performed by the unweighted pair-group mean analysis (UPGMA).
FIGURE 2

Pulsed field gel electrophoresis of Ralstonia mannitolilytica isolated from patients and Vapotherm devices, Israel, JuneJuly $2011(n=10)$

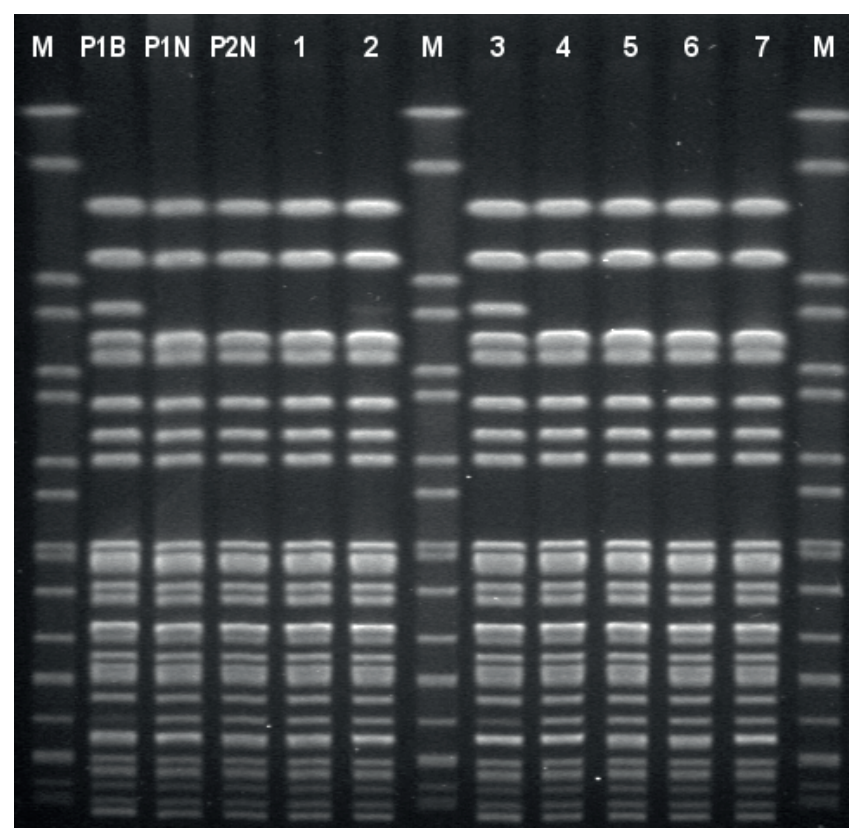

M: Size marker; $\mathrm{P}_{1} \mathrm{~B}$ : blood culture isolate from index case; $\mathrm{P}_{1} \mathrm{~N}$ : nasal isolate from index case; $\mathrm{P}_{2} \mathrm{~N}$ : nasal isolate from second infant; 1 : isolate from gas line of delivery tube; 2 : isolate from water jacket of delivery tube; 3-6: isolates from four used vapour transfer cartridges from the Mount Scopus hospital; 7 : isolate from a used vapour transfer cartridge from the Ein Kerem hospital.

\section{Results}

\section{Microbiological investigation}

The isolates recovered from the index patient's blood cultures were oxidase-positive and colistin-resistant. All had an API 20NE profile at $48 \mathrm{~h}$ of 0045555 , giving low probability identifications of Pseudomonas fluorescens or Ralstonia pickettii, with Ochrobactrum anthropi as an additional possibility. BLAST analysis of a 730 bp 16SrRNA gene sequence revealed that the isolate was a Ralstonia species, most similar to $R$. mannitolilytica, and the organism was indeed mannitol-positive. This species had not previously been identified at our hospitals. Subsequently, the identity of the isolates was further confirmed by MALDI-TOF mass spectrometry (Vitek MS, BioMerieux, Marcy l'Etoile, France) as R. mannitolilytica (99.9\%).

Nine of the 15 patients including the index case had been treated with the Vapotherm 200oi at different times during their hospital stays. $R$. mannitolilytica was isolated from the blood and nose of the index case and the nose of one additional child who had been treated with the device (each child had their own cartridge and 
tubing, which were kept until the treatment course was completed). In addition to the three patient isolates, $R$. mannitolilytica was obtained from seven environmental samples: from the index patient's delivery tube (both from the gas line and the water jacket), from all four vapour transfer cartridges from Mount Scopus and from one of the two from Ein Kerem. Culture of the unopened cartridge and of the sterile water supplied by the hospital was negative. No bacterial DNA was recovered from the contents of the remaining two unopened cartridges (of the same batch).

Eight of the 10 patient and environmental isolates from the two hospitals were found identical by PFGE (Figure 2) using accepted criteria [16], while two showed the same single-band difference, namely one of the two isolates from the index patient and one of the cartridge isolates from Mount Scopus.

\section{Comparison with the 2005}

\section{outbreak in the United States}

Apart from the use of an unfiltered instead of a filtered sterile needle to vent the sterile water containers, no significant deviations from the instructions were identified in the reported reprocessing procedure. Examination of the dates of manufacture of the cartridges revealed that at least two of the recently used lots were manufactured in October 2005, before the recall of the system in the US.

Inspection of the PFGE image published in the report of the 2005 US outbreak suggested some similarity between our isolates and those of the US outbreak [1] (Figure 3). Since standardised PulseNet protocols had been used in both investigations, a comparison was therefore undertaken by the Israel Ministry of Health Molecular Biology Laboratory in collaboration with investigators at the CDC who had studied the US event. The comparison included 16 representative US 2005 outbreak-associated isolates and four unassociated isolates. The analysis showed that the eight identical isolates from Israel 2011 were indistinguishable from the US 2005 outbreak isolates, which originated from 22 hospitals in 13 US States, while the two single-band variants showed more than $97 \%$ similarity with these (Figure 3). Those isolates in the US study that were unassociated with the outbreak were unrelated, with less than $80 \%$ similarity.

\section{Discussion}

We have described a cluster of $R$. mannitolilytica isolates that were detected following a bloodstream infection in a premature infant, associated with colonisation of an additional patient and contamination of components of the Vapotherm 200oi device. The outstanding finding of this study was the possibility that the same organism was responsible for two events of contamination separated geographically and temporally by several years (Figure 4).
Although no final conclusion was reached in the US investigation as to the origin of the organism, our combined data strongly suggest that the organisms isolated from both events had the same source. Nevertheless, the question as to what this source was will probably remain unresolved.

While in the CDC investigation in 2005, $R$. mannitolilytica was not isolated from 26 unopened vapour transfer cartridges representing 13 lots, the organism was isolated from such cartridges at two US hospitals [1]. At one hospital, $R$. mannitolilytica was recovered from three unused cartridges from the same lot; at the other, it was grown from three of 10 unused cartridges from different lots [1].

The phenomenon of the reappearance in a different country of a single strain of such a clinically rare organism, in association with the same equipment and after several years, refocuses on the possibility that some cartridges may have been contaminated, perhaps during a calibration process during manufacture that was referred to in the US report. This process used tap water, which suggested that the cartridges might be the leading candidate as a potential source. Cultures of tap water from the Irish plant that calibrated the cartridges, performed at the CDC during that investigation, did not confirm this [1].

The apparent tenacity of the organism is also a testament to the survival capacity of this strain, or perhaps even the species, which might spur further investigation of this ability. An incentive to study this phenomenon was provided by an informal observation in our laboratory. Colonies of our isolates of $R$. mannitolilytica usually survived no longer than seven or eight days on culture plates, whereas subcultures from suspensions in sterile water maintained at room temperature for over six months produced rapid rich growth of the organism.

To explain the incident in Jerusalem, understanding the solution of the problem adopted in the US in 2006-07 is crucial. The measures introduced included two key changes regarding the cartridges, i) sterilisation of the cartridges during manufacture to ensure their sterility prior to use and ii) discarding the cartridges before decontamination of the water and gas circuits (i.e. single use only, with no reprocessing) $[1,17]$. These steps would ensure that if contamination of the cartridges were either intrinsic to their manufacture or their calibration, or occurred by some process during use, the chances of transmission of the organism to one or more patients would be minimised or eliminated. Neither of these conditions was met in the instructions provided to us by the local distributor in Israel.

Furthermore, as part of the US solution, the kit provided for routine cleaning (CK-101) before the outbreak, which included disinfection of the cartridge, was replaced by a new kit for disinfection (DK-301) that was 
Dendrogram of pulsed-field gel electrophoresis results showing per cent similarity of Ralstonia mannitolilytica isolates from the United States in $2005(\mathrm{n}=20)$ and from Israel in $2011(\mathrm{n}=10)$
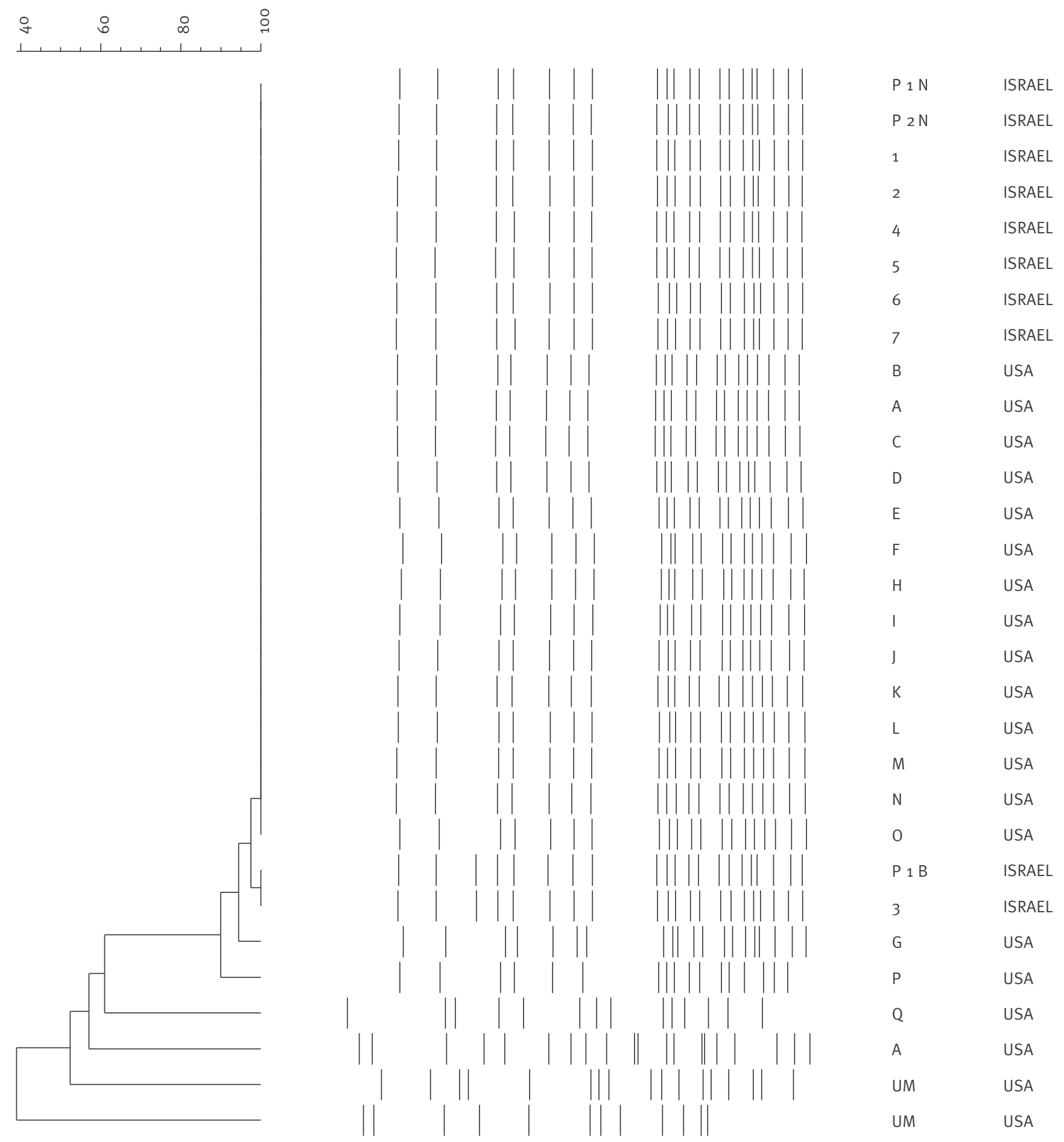

$\mathrm{P}_{1} \mathrm{~N}$ : index case, nasal isolate; $\mathrm{P}_{2} \mathrm{~N}$ : second infant, nasal isolate; $\mathrm{P}_{1} \mathrm{~B}$ : index case, blood culture isolate; 1-7: environmental isolates from the present investigation. All other isolates were from the United States: those with greater than $80 \%$ similarity were associated with the 2005 outbreak and those with less than $80 \%$ similarity were unrelated strains isolated previously, including two reference strains from the University of Michigan (UM). 
Timeline of the Ralstonia mannitolilytica events in the United States, 2005, and in Israel, 2011

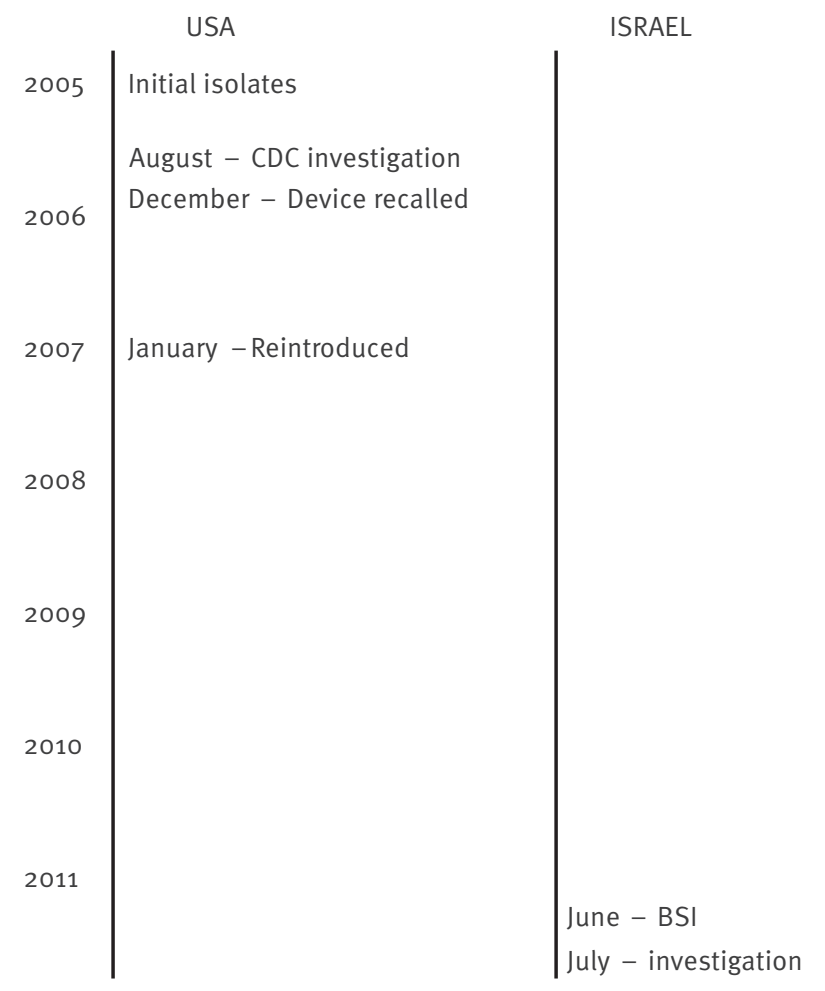

BSI: Bloodstream infection of the index patient.

intended for use after discarding the single use cartridge and bridging the resulting gap in the tubing with the cartridge bypass tube provided in the DK-301 kit. This latter kit was, to the best of our knowledge, not made available to Israeli customers, although the local distributor offered an annual high-level disinfection service for which the DK-301 would be deployed (information provided by the local company and the manufacturer). The instructions provided to our institution included reprocessing the cartridges with the proprietary solution of a mix of quaternary ammonium compounds provided with the CK-101 cleaning kit (Control III, Maril Products Inc., Tustin, CA, US). US instructions for use of the Vapotherm 2000i after 2006 state that this solution is not approved for cartridge disinfection [18].

The unopened cartridges we examined for growth or for bacterial DNA (VTo1-B type) were produced in 2005. While they bore the CE mark, they were neither marked as sterile, nor for single use. The containers of the cartridges that had been in use and that were manufactured in 2005, 2009 and 2010, including those with positive cultures, were not available for inspection in this regard.
After reintroduction of the device in the US in 2007, customers were invited to have their old VTo1-A and VTo1-B cartridges replaced by the new devices VTo1-AS and VT01-BS as specified in a company document [19] and the associated FDA document [20].

The exact time and manner of introduction of $R$. mannitolilytica in our institution cannot be determined. This was the first time that this taxon was identified at our laboratory. Our data, with the corroborative comparison with the US isolates, indicate that the organism isolated at both our hospitals probably had its source in one or more items of equipment used in the Vapotherm 200oi device. In view of the extremely high similarity of the isolates from our two hospitals and from the US event, we consider it unlikely that venting the sterile water containers with unfiltered needles were responsible for the problem.

Users of the Vapotherm 200oi system should be aware that reprocessing of cartridges regardless of the disinfectant used should not be practiced as this might constitute a possible risk of infection. It should be remembered that our situation was revealed by a bloodstream infection in a premature infant. This raises the question of the desirability of microbiological checks of potentially hazardous equipment or procedures.

In a commentary on the CDC investigation of the US outbreak, Saiman emphasised the importance of correctly classifying patient care equipment in terms of the level of disinfection required to keep that equipment safe for use on patients [21]. It is regrettable that this principle, so admirably applied in the US, does not seem to have been promoted elsewhere.

\section{Acknowledgements}

The authors gratefully acknowledge the assistance and expert contribution of Dr Judith Noble-Wang and Dr Matthew J. Arduino of the Centers for Disease Control and Prevention, Atlanta, GA, United States.

Conflict of interest

None declared. 


\section{References}

1. Jhung MA, Sunenshine RH, Noble-Wang J, Coffin SE, St John K, Lewis FM, et al. A National Outbreak of Ralstonia mannitolilytica Associated With Use of a Contaminated Oxygen-Delivery Device Among Pediatric Patients. Pediatrics. 2007:119(6):1061-8.

http://dx.doi.org/10.1542/peds.2006-3739 PMid:17545371

2. Yabuuchi E, Kosako Y, Yano I, Hotta H, Nishiuchi Y. Transfer of two Burkholderia and an Alcaligenes species to Ralstonia gen. Nov.: Proposal of Ralstonia pickettii (Ralston, Palleroni and Doudoroff 1973) comb. Nov., Ralstonia solanacearum (Smith 1896) comb. Nov. and Ralstonia eutropha (Davis 1969) comb. Nov. Microbiol Immunol. 1995;39(11):897-904. PMid:8657018

3. Penna V, Martins S, Mazzola P. Identification of bacteria in drinking and purified water during the monitoring of a typical water purification system. BMC Public Health. 2002;2:13. http://dx.doi.org/10.1186/1471-2458-2-13 PMid:12182763 PMCid:122092

4. Barbut F, Kosmann M-I, Lalande V, Neyme D, Coppo P, Gorin NC. Outbreak of Ralstonia pickettii Pseudobacteremia Among Patients With Hematological Malignancies. Infect Control Hosp Epidemiol. 2006;27(6):642-4. http://dx.doi. org/10.1086/505100 PMid:16755490

5. Degand N, Carbonnelle E, Dauphin B, Beretti JL, Le Bourgeois M, Sermet-Gaudelus I, et al. Matrix-Assisted Laser Desorption Ionization-Time of Flight Mass Spectrometry for Identification of Nonfermenting Gram-Negative Bacilli Isolated from Cystic Fibrosis Patients. J Clin Microbiol. 2008;46(10):3361-7. http://dx.doi.org/10.1128/JCM.00569-08 PMid:18685005 PMCid:2566097

6. Coenye T, Vandamme P, LiPuma JJ. Infection by Ralstonia Species in Cystic Fibrosis Patients: Identification of R. pickettii and R. mannitolilytica by Polymerase Chain Reaction. Emerg Infect Dis. 2002;8(7):692-6. http://dx.doi.org/10.3201/ eid0807.010472 PMid:12095436 PMCid:2730328

7. De Baere T, Steyaert S, Wauters G, Des Vos P, Goris J, Coenye T, et al. Classification of Ralstonia pickettii biovar 3/'thomasii' strains (Pickett 19.4) and of new isolates related to nosocomial recurrent meningitis as Ralstonia mannitolytica sp. nov. Int J Syst Evol Microbiol. 2001;51(2):547-58. PMid:11321101

8. Centers for Disease Control and Prevention. Ralstonia associated with Vapotherm oxygen delivery device--United States, 2005. MMWR Morb Mortal Wkly Rep. 2005;54(41):10523. PMid:16237377

9. Gröbner S, Heeg P, Autenrieth IB, Schulte B. Monoclonal outbreak of catheter-related bacteraemia by Ralstonia mannitolilytica on two haemato-oncology wards. J Infect 2007;55(6):539-44. http://dx.doi.org/10.1016/j.jinf.2007.07.021 PMid:17881058

10. Daxboeck F, Stadler M, Assadian O, Marko E, Hirschl AM, Koller W. Characterization of clinically isolated Ralstonia mannitolilytica strains using random amplification of polymorphic DNA (RAPD) typing and antimicrobial sensitivity, and comparison of the classification efficacy of phenotypic and genotypic assays. J Med Microbiol 2005;54(Pt1):55-61. http://dx.doi.org/10.1099/jmm.0.45656-0 PMid:15591256

11. Coenye T, Vandamme P, LiPuma JJ. Infection by Ralstonia species in cystic fibrosis patients: identification of R. pickettii and R. mannitolilytica by polymerase chain reaction. Emerg Infect Dis. 2002;8(7):692-6. http://dx.doi.org/10.3201/ eido807.010472 PMid:12095436 PMCid:2730328

12. Vaneechoutte M, De Baere T, Wauters G, Steyaert S, Claeys G Vogelaers D, et al. One Case Each of Recurrent Meningitis and Hemoperitoneum Infection with Ralstonia mannitolilytica. J Clin Microbiol. 2001;39(12):4588-90. http://dx.doi.org/10.1128/ JCM.39.12.4588-4590.2001 PMid:11724893 PMCid:88597

13. Centers for Disease Control and Prevention. Update: Ralstonia species associated with Vapotherm oxygen delivery devices--United States, 2005. MMWR Morb Mortal Wkly Rep. 2005:54(43):1104-5. PMid:16267498

14. Chakravorty S, Helb D, Burday M, Connell N, Alland D. A detailed analysis of $16 \mathrm{~S}$ ribosomal RNA gene segments for the diagnosis of pathogenic bacteria. J Microbiol Methods 2007;69(2):330-9. http://dx.doi.org/10.1016/j. mimet.2007.02.005 PMid:17391789 PMCid:2562909

15. Ribot EM, Fair MA, Gautom R, Cameron DN, Hunter SB, Swaminathan B, et al. Standardization of Pulsed-Field Gel Electrophoresis Protocols for the Subtyping of Escherichia coli 0157:H7, Salmonella, and Shigella for PulseNet. Foodborne Pathog Dis 2006;3(1):59-67. http://dx.doi.org/10.1089/fpd.2006.3.59 PMid:16602980

16. Tenover FC, Arbeit RD, Goering RV, Mickelsen PA, Murray BE, Persing DH, et al. Interpreting chromosomal DNA restriction patterns produced by pulsed-field gel electrophoresis: criteria for bacterial strain typing. J Clin Microbiol. 1995;33(9):2233-9. PMid:7494007 PMCid:228385

17. Centers for Disease Control and Prevention. Update: Ralstonia apecies aontamination associated with Vapotherm ${ }^{\circledR} 2000 \mathrm{i}$ respiratory gas humidifier systems -.- United States, 2005-2006. MMWR Morb Mortal Wkly Rep. 2007;56(8):173.

18. Vapotherm 200oi Operating instruction manual, Rev. E. Stevensville: Vapotherm Inc. [Accessed 29 December 2012]. Available from: http://www.vtherm.com/_pdfs/Operating\%20 Manual_2000i_RevE\%2006.pdf

19. Vapotherm 200oi post recall frequently asked questions. Stevensville: Vapotherm Inc. [Accessed 29 December 2012] Available from: http://www.vtherm.com/_pdfs/FAQ\%2ofor\%20 the \%20Website\%20_12-8-06\%20track\%20changes_\%20_2_. pdf

20. FDA Public Health Notification: Precautions in Using the Reintroduced Vapotherm ${ }^{\circledR}>2000$ [ [Respiratory Gas Humidifier] System. Silver Spring: Unites States Food and Drug Administration. [Accessed 29 December 2012. Available from: http://www.fda.gov/MedicalDevices/Safety/AlertsandNotices/ PublicHealthNotifications/UCM062083

21. Saiman L. Providing a Safety Net for Children: Investigating a Multistate Outbreak of Ralstonia mannitolilytica Related to a Contaminated Reusable Device. Pediatrics 2007;119(6):1207-9. http://dx.doi.org/10.1542/peds.2007-0787 PMid:17545391 